Original Research Paper

\title{
Finite Element Formulation for Active Composite Laminates
}

\author{
${ }^{1,2}$ Dragan Marinkovic and ${ }^{1}$ Manfred Zehn \\ ${ }^{I}$ Department of Structural Analysis, Berlin Institute of Technology, Berlin, Germany \\ ${ }^{2}$ Faculty of Mechanical Engineering, University of Nis, Nis, Serbia
}

Article history

Received: 21-04-2015

Revised: 04-05-2015

Accepted: 29-05-2015

Corresponding Author: Dragan Marinkovic

Department of Structural

Analysis, Berlin Institute of

Technology, Berlin, Germany

Email: gagimarinkovic@yahoo.com

\begin{abstract}
Smart structures are characterized by a synergistic integration of active materials into a passive structure connected by a control system to enable an automatic adaptation to changing environmental conditions. Piezoelectric materials are widely used as distributed sensors and actuators in smart structures, where especially hybrid composites - a combination of fiber-reinforced and piezoelectric laminae - are very powerful smart material systems. First, a brief review of the developed shell type finite element for smart composite structures is presented. It is a degenerated shell element based on the Reissner-Mindlin kinematical assumptions for modeling thin and moderately thick structures made of multilayered material including piezoelectric active layers polarized in the thickness direction. The main focus of the paper is put on the test examples originally proposed by other authors. The finite element results were compared with the reference solutions obtained by Ritz type approximations. The considered test cases investigate the effects of shell shallowness, bending/twisting coupling and the influence of the piezoelectric layer thickness on the deformation of the laminated structure.
\end{abstract}

Keywords: Smart Structures, Finite Element, Shell, Piezo, Composite

\section{Introduction}

A synergic integration of structures, multi-functional materials based sensors and actuators and control electronics has redefined the concept of structures from a conventional passive elastic system to an active controllable system with inherent self-sensing, diagnosis, control and actuation capabilities. Such integration enables the structure to respond in real time or nearly real time to external stimuli to compensate for undesired behavior or to produce a desired response through the change of the structure's stiffness, inertial properties, damping properties or configuration. A new name emerged: Active or "smart" structures. The paper considers an important and growing group of active structures, whose areas of application become more diverse each day. Those are hybrid composite thin-walled structures with piezoelectric material based active components and fiber-reinforced composite laminates as a passive material.

The potential benefits the active structures offer over the classical "passive" structures attracted many researchers from various fields to this new multidisciplinary field. The development and design of active structures requires reliable, accurate and numerically efficient tools for their modeling and simulation. At the present stadium of development the Finite Element Method (FEM) as a predominant one is almost inevitably addressed on this matter. The body of literature considering the finite element approach to modeling active structures is quite large and an interested reader is referred to surveys such as the one from Benjeddou (2000). This paper presents a part of the authors' contribution to modeling and simulation of the behavior of the considered structures. It gives a brief description of the developed shell element (Marinkovic, 2007) that has been already tested for passive linear cases (Marinkovic et al., 2012) as well as for passive and active geometrically linear and nonlinear cases (Marinkovic et al., 2013; 2008). The developed element has been recently implemented in commercially available FEM program ABAQUS in order to facilitate its application (Nestorovic et al., 2013; 2014).

\section{Formulation of a Shell Element for Piezoelectric Composite Laminates}

The finite element modeling of a generally shaped thin-walled active structure requires the application of a 
shell type finite element. Among different approaches to finite element modeling of shell type structures the degenerated shell approach is the most promising one. The degenerated shell element was first developed by Ahmad et al. (1970) from a 3D solid element by a degeneration process, which directly reduced the $3 \mathrm{D}$ field approach to a 2D one in terms of mid-surface nodal variables. The most significant advantages achieved in this manner are that the element is not based on the classical shell theories and is applicable over a wide range of thickness and curvatures. The developed element, referred to as 9-node active composite shell (ACShell9) represents the extension to modeling active multi-layered structures made of fiber-reinforced composites and including active piezoelectric layers polarized in the thickness direction.

\section{The Element Geometry and Mechanical Field}

Due to the complexity of the degenerate shell element several coordinate systems are needed to describe the element geometry, displacement field and strain field. Besides the global $(x, y, z)$ and the natural $(r, s, t)$ coordinate system, it is necessary to introduce a local coordinate system $\left(x^{\prime}, y^{\prime}, z^{\prime}\right)$, Fig. 1b. The local coordinate system is defined so as to have one of its axes (say $z$-axis) perpendicular to the mid-surface, while the other two axes form the tangential plane. The kind of nonisotropy exhibited by the fiber-reinforced composite laminates requires introduction of a structure reference direction (defined by the user), with respect to which the fiber orientation in the layers is given. In this case it is reasonable to fix the orientation of the local in-plane axes with respect to the structure reference direction. The simplest way is overlapping one of the axes (say $x^{\prime}$-axis) with it (Fig. 1a).

Using the full biquadratic Lagrange shape functions $\mathrm{N}_{\mathrm{i}}$ (Marinkovic, 2007), the coordinates of a mid-surface point are given by Equation 1:

$$
\left\{\begin{array}{lll}
x & y & z
\end{array}\right\}^{T}=\sum_{i=1}^{9} N_{i}\left\{\begin{array}{lll}
x_{i} & y_{i} & z_{i}
\end{array}\right\}^{T}
$$

With $x_{i}, y_{i}$ and $z_{i}$ denoting the global coordinates of the nine nodes. The thickness of the shell is assumed to be in the direction normal to the mid-surface. Denoting the unity vectors of the local coordinate system with respect to the global coordinate system by $\vec{e}_{a i}$, where $a$ stands for $x^{\prime}, y^{\prime}$ or $z^{\prime}$ depending on the axis and $i$ denotes the node, the $3 \mathrm{D}$ shell geometry may be regenerated from its mid-surface in the following way Equation 2:

$$
\left\{\begin{array}{lll}
x & y & z
\end{array}\right\}^{T}=\sum_{i=1}^{9} N_{i}\left\{\begin{array}{lll}
x_{i} & y_{i} & z_{i}
\end{array}\right\}^{T}+\sum_{i=1}^{9} \frac{h_{i}}{2} N_{i} t \vec{e}_{z^{\prime} i}^{T}
$$

where, $h_{i}$ denotes the shell thickness at node $i$ and the natural thickness coordinate takes values: $-1<\mathrm{t}<+1$.

The degeneration process is based on the assumption that the thickness direction line of the shell remains straight after deformation but not necessarily perpendicular to the mid-surface (the Mindlin kinematical assumption). Therefore, the displacement of any point within the volume of the shell is given as a superposition of the corresponding mid-surface point displacement and a linear function of the rotations about the local $x^{\prime}$ - and $y$ '-axis through the midsurface point, $\theta_{x}$, and $\theta_{y}$, Equation 3:

$$
\begin{aligned}
& \left\{\begin{array}{lll}
u & v & w
\end{array}\right\}^{T}= \\
& =\sum_{i=1}^{9} N_{i}\left\{\begin{array}{lll}
u_{i} & v_{i} & w_{i}
\end{array}\right\}^{T}+\sum_{i=1}^{9} \frac{h_{i}}{2} N_{i} t\left[\begin{array}{ll}
-\vec{e}_{y^{\prime} i} & \vec{e}_{x^{\prime} i}
\end{array}\right]\left\{\begin{array}{ll}
\theta_{x^{\prime}} & \theta_{y^{\prime}}
\end{array}\right\}^{T}
\end{aligned}
$$

The rotations are in the local coordinate system and upon transformation to the global c.s. one gets:

$$
\begin{aligned}
& \left\{\begin{array}{lll}
u & v & w
\end{array}\right\}^{T}=\sum_{i=1}^{9} N_{i}\left\{\begin{array}{lll}
u_{i} & v_{i} & w_{i}
\end{array}\right\}^{T}+ \\
& +\sum_{i=1}^{9} \frac{h_{i}}{2} N_{i} t\left[\begin{array}{ll}
-\vec{e}_{y^{\prime} i} & \vec{e}_{x^{\prime} i}
\end{array}\right]\left[\begin{array}{lll}
T^{\prime}
\end{array}\right]^{T}\left\{\begin{array}{lll}
\theta_{x} & \theta_{y} & \theta_{z}
\end{array}\right\}^{T}
\end{aligned}
$$

where, $\left[T^{\prime}\right]$ is the modified form of the transformation matrix relating the local and the global coordinate system, $[T]$ and is given in the form $\left[T^{\prime}\right]=\left[\begin{array}{ll}\vec{e}_{x^{\prime}} & \vec{e}_{y^{\prime}}\end{array}\right]$.

Due to the directionally dependent material properties, it is of crucial importance to develop the strain field in the local coordinate system. This allows direct application of the composite laminates constitutive matrix, the so-called ABD matrix. The advantage of having the strain field with respect to the local coordinate system is also obvious when the piezoelectric coupling within the thickness-polarized piezopatch operating on the " $\mathrm{e}_{31}$-effect" is considered.

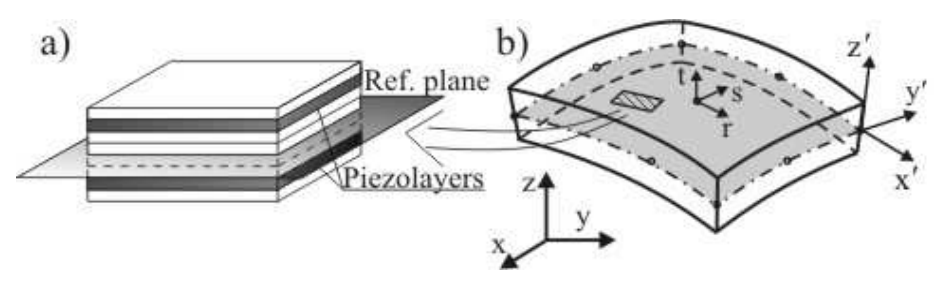

Fig. 1. Element geometry 
The interpolations are performed in the natural c.s. Hence, the displacement derivatives with respect to the natural coordinates are directly obtained from Equation 4. The transformation of derivatives from the natural to the global coordinate system is achieved by means of Jacobian inverse Equation 5:

$$
\begin{aligned}
& {[J]=\left[\begin{array}{lll}
x,_{r} & y,_{r} & z,,_{r} \\
x,_{s} & y,_{,_{s}} & z,,_{s} \\
x,_{t} & y,_{t} & z,_{t}
\end{array}\right] \Rightarrow} \\
& {\left[\begin{array}{lll}
u,_{x} & v,_{x} & w,_{x} \\
u,_{y} & v,_{y} & w,_{y} \\
u,_{z} & v,_{z} & w,_{z}
\end{array}\right]=[J]^{-1}\left[\begin{array}{lll}
u,_{r} & v,_{r} & w,_{r} \\
u,_{s} & v,_{s} & w,_{s} \\
u,_{t} & v,_{t} & w,_{t}
\end{array}\right]}
\end{aligned}
$$

where, for example, $u,_{x}=\partial u / \partial x$. The global derivatives are afterwards transformed to the local derivatives by means of the transformation matrix $[T]=\left[\begin{array}{lll}\vec{e}_{x^{\prime}} & \vec{e}_{y^{\prime}} & \vec{e}_{z^{\prime}}\end{array}\right]$ :

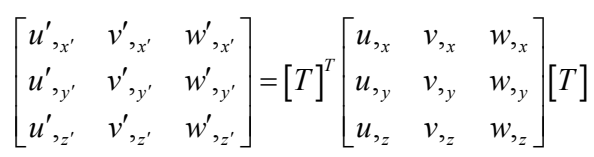

It is quite common within a $2 \mathrm{D}$ formulation to give the strain field in the form that makes a distinction between the in-plane components and the out-of-plane strain components Equation 7:

$$
\begin{aligned}
& \left\{\varepsilon^{\prime}\right\}=\left\{\begin{array}{llllll}
\varepsilon_{x^{\prime} x^{\prime}} & \varepsilon_{y^{\prime} y^{\prime}} & \gamma_{x^{\prime} y^{\prime}} & \text { i } & \gamma_{y^{\prime} z^{\prime}} & \gamma_{x^{\prime} z^{\prime}}
\end{array}\right\}^{T} \\
& \text { i.e. }
\end{aligned}
$$

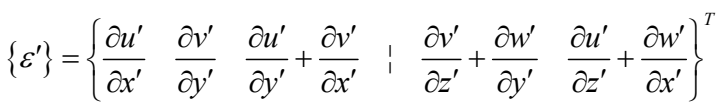

After determining the partial derivatives in the local coordinate system (Equation 6), the strain field is given in the following form (more details about the procedure in (Marinkovic, 2007) Equation 8:

$$
\begin{aligned}
& \left\{\varepsilon^{\prime}\right\}=\left\{\begin{array}{c}
\left\{\varepsilon_{m f}^{\prime}\right\} \\
-- \\
\left\{\varepsilon_{s}^{\prime}\right\}
\end{array}\right\}=\left[\begin{array}{c}
{\left[B_{m f}\right]} \\
-- \\
{\left[B_{s}\right]}
\end{array}\right]\{d\} \\
& =\left[\begin{array}{ccc}
{\left[B_{T m}\right]} & t\left[B_{R 1 f}\right] \\
-- & + & ------- \\
{\left[B_{T s}\right]} & : & {\left[B_{R 0 s}\right]+t\left[B_{R 1 s}\right]}
\end{array}\right]\{d\}=\left[B_{u}\right]\{d\}
\end{aligned}
$$

where, $\left\{\varepsilon_{m f}^{\prime}\right\}$ is the membrane-flexural (in-plane) strain field, $\left\{\varepsilon_{s}^{\prime}\right\}$ comprises the transverse shear strains, $\left[B_{m f}\right]$ and $\left[B_{s}\right]$ are the corresponding strain-displacement matrices further suitably represented in terms of the B- matrices having " $m$ ", " $f$ " and " $s$ " in the subscript depending whether they contribute to the definition of the membrane, flexural or shear strains, respectively, those having " $T$ " are related to the nodal translations and with " $R$ " are related to the nodal rotations and finally, " 0 " denotes constant terms while " 1 " denotes linear terms with respect to the natural thickness coordinate $t$. The vector $\{d\}$ comprises nodal displacements (translations and rotations).

\section{Piezoelectric Layers}

The constitutive equations of the piezoelectric material depend on the choice of the independent variables. Within the FE analysis it is suitable to choose the mechanical strain and the electric field, so that:

$$
\begin{aligned}
& \{\sigma\}=\left[C^{E}\right]\{\varepsilon\}-[e]\{E\} \\
& \{D\}=[e]\{\varepsilon\}+\left[d^{\varepsilon}\right]\{E\}
\end{aligned}
$$

where, $\{\sigma\}$ is the mechanical stress in vector (Voigt) notation, $\{D\}$ is the electric displacement vector, $\left[C^{E}\right]$ is the piezoelectric material Hook's matrix at constant electric field $E,\left[d^{\varepsilon}\right]$ is the dielectric permittivity matrix at $\varepsilon$ constant and $[e]$ is the piezoelectric coupling matrix.

This paper considers piezoceramic elements with electrodes on the top and bottom surfaces and poled in the thickness direction, where the in-plane strains are coupled with the perpendicularly applied electric field through the piezoelectric " $\mathrm{e}_{31}$-effect". The typical approximations yield a linear distribution of the electric field and a constant electric field through the piezolayers thickness. They are adopted here, thus Equation 10:

$$
E=-\frac{\partial \phi}{\partial z^{\prime}} \Rightarrow E_{k}=-\frac{\Delta \Phi_{k}}{h_{k}}
$$

where, $\varphi$ is the electric potential, $\Delta \Phi_{k}$ is the difference of the electric potentials between the electrodes of the $\mathrm{k}^{\text {th }}$ layer and $h_{k}$ is the thickness of the piezolayer. The approximation in the Equation 9 defines a diagonal electric field-electric potential matrix $\left[B_{\phi}\right]$ with typical term $1 / h_{k}$ on the main diagonal. The diagonal form of the matrix $\left[B_{\phi}\right]$ results from the fact that the difference of the electric potentials of a layer affects only the electric field within the very same layer.

\section{Finite Element Equations}

The finite element equations for dynamic cases involving a piezoelectric continuum are obtained using the variational approach (Marinkovic, 2007) and they are given in the following standard form: 


$$
\begin{aligned}
& {[M]\{\ddot{d}\}+[C]\{\dot{d}\}+\left[K_{u u}\right]\{d\}+\left[K_{u \varphi}\right]\{\varphi\}=\left\{F_{\text {ext }}\right\}} \\
& {\left[K_{\varphi u}\right]\{d\}+\left[K_{\varphi \varphi}\right]\{\varphi\}=\left\{Q_{e x t}\right\}}
\end{aligned}
$$

where, the following matrices and vectors are introduced on the element level: The mass $[M]$, (proportional Rayleigh) damping $[C]$, mechanical stiffness $\left[K_{u u}\right]$, piezoelectric stiffness $\left[K_{u \phi}\right]=\left[K_{\phi u}\right]^{T}$ and dielectric stiffness $\left[K_{\phi \phi}\right]$ matrices as well as vectors of external forces $\left\{F_{\text {ext }}\right\}$ and electric charges $\left\{Q_{\text {ext }}\right\}$. Although Equation 12 appears to be static (no obvious dynamic effects included), it should be noted that it is coupled to the Equation 11 that contains dynamic mechanical effects. The system of equations for a static case is obtained in a straightforward manner by excluding dynamic effects, i.e. inertia and damping, from Equation 11.

\section{Numerical Examples and Discussion}

In the following, three static examples will be considered. The examples are originally proposed by Kioua and Mirza (2000) and solved by using the conventional Ritz analysis based on the shallow-shell approximations. The finite element results also include those yielded by the Semi-Loof active shell element (Seeger et al., 2001).

In all three cases the considered structures have dimensions $\mathrm{a} \times \mathrm{b}=254 \times 254 \mathrm{~mm}$. They are made of hybrid composite laminates, the outer layers of which are made of PZT G1195 piezoceramic (thickness 0.254 $\mathrm{mm}$ ) and the internal layers are of T300/976 graphite/epoxy (thickness $0.138 \mathrm{~mm}$ ). The properties of the layers are given in Table 1 . The sequence of the internal layers will differ from case to case and will be specified separately for each case. The piezoelectric constants that are not specified here are considered to be equal to zero. In all cases the piezoelectric excitation is achieved by supplying the same voltage to the oppositely polarized piezolayers, which results in bending moments uniformly distributed over the edges of the structure. Thus, only the actuator function of the piezolayers is considered and the dielectric constants are therefore not necessary for the calculation purposes and are not given in Table 1.

\section{Shape Control of an Active Plate}

A simply supported cross-ply plate, with the internal sequence of layers $[0 / 90 / 0]_{S}$, is initially subjected to a uniformly distributed load of $200 \mathrm{~N} / \mathrm{m}^{2}$. Then the gradually increasing voltage is supplied to the outer, active layers, with the aim of finding the voltage giving the shape that corresponds mostly to the initial

\begin{tabular}{|c|c|c|}
\hline Material properties & $\begin{array}{l}\text { PZT G1195 } \\
\text { piezolayer }\end{array}$ & $\begin{array}{l}\text { T300/976 } \\
\text { graphite/epoxy }\end{array}$ \\
\hline \multicolumn{3}{|l|}{ Elastic properties } \\
\hline $\mathrm{Y}_{11}(\mathrm{GPa})$ & 63.0 & 150.0 \\
\hline $\mathrm{Y}_{22}(\mathrm{GPa})$ & 63.0 & 9.0 \\
\hline $\mathrm{v}_{12}$ & 0.3 & 0.3 \\
\hline $\mathrm{G}_{12}(\mathrm{GPa})$ & 24.2 & 7.1 \\
\hline \multicolumn{3}{|l|}{ Piezoelectric properties } \\
\hline $\mathrm{e}_{31}\left(\mathrm{C} / \mathrm{mm}^{2}\right)$ & $2.286 \cdot 10^{-5}$ & 0.0 \\
\hline $\mathrm{e}_{32}\left(\mathrm{C} / \mathrm{mm}^{2}\right)$ & $2.286 \cdot 10^{-5}$ & 0.0 \\
\hline
\end{tabular}
undeformed shape.
An $8 \times 8$ finite element mesh is applied and the normalized center line deflection is calculated for the same voltages as given in the paper by Kioua and Mirza (2000), i.e., $0 \mathrm{~V}$ (initial deformed shape under distributed load), 15 and $27 \mathrm{~V}$. Figure 3 shows the results obtained with the ACShell9 element and the Semi-Loof element (solid lines, nearly congruent) and the results from Kioua and Mirza (dashed lines). According to the Ritz solution, the structure recovers the flat shape for the last applied voltage (Fig. 2, 27 V). However, the FEM results show that the structure subjected to the voltage of $27 \mathrm{~V}$ (and the uniform load) is not exactly flat, although very close to it. It should be noted that the act of the moments uniformly distributed over the plate edges (obtained by the actuation of the piezolayers) certainly cannot recover the original (unloaded) flat geometry.

\section{Clamped Cylindrical Shell}

A cantilevered cylindrical shell is considered in the second case (Fig. 3). Fiber-reinforced composite layers have the sequence $\left[30_{2} / 0\right]_{\mathrm{s}}$. Obviously, the sequence is unbalanced resulting in coupling between bending and twisting. The same voltage of $\varphi=100 \mathrm{~V}$ is supplied to outer (piezo) layers. Since both bending and twisting of the shell are induced, the transverse deflection is observed at three characteristic points, two end-points and the mid-point of the free edge, denoted in Fig. 3 as 1,3 and 2, respectively.

The same mesh of $8 \times 8$ elements is used for the finite element results. The different radii $\mathrm{R}$ of the curvature are considered. As an indicator of bending deformation, the ratio $\left(\mathrm{w}_{2} / \mathrm{b}\right)$ is observed, while the ratio $\left(\mathrm{w}_{3}-\mathrm{w}_{1}\right) / \mathrm{b}$ is taken as an indicator of twisting. The comparison of the results is given in Fig. 4. A very high agreement of the results from the ACShelly and the Semi-Loof element can be noted. The Ritz solution is also close to the FE results, especially for higher values of radius to span ratio, which is expectable. As this ratio takes lower values, the shell behavior becomes more complex and cannot be accurately enough described by relatively simple shape functions assumed within the presented Ritz solution. 


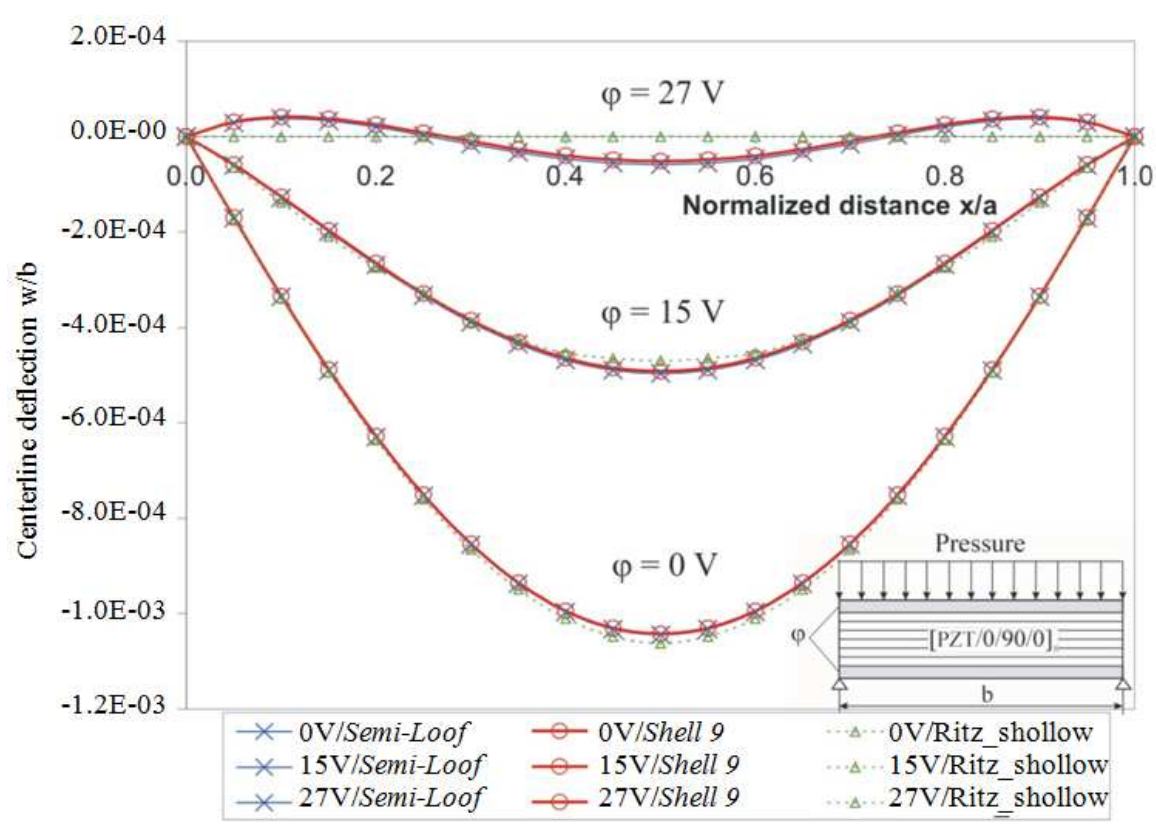

Fig. 2. Shape control of the simply supported composite active plate

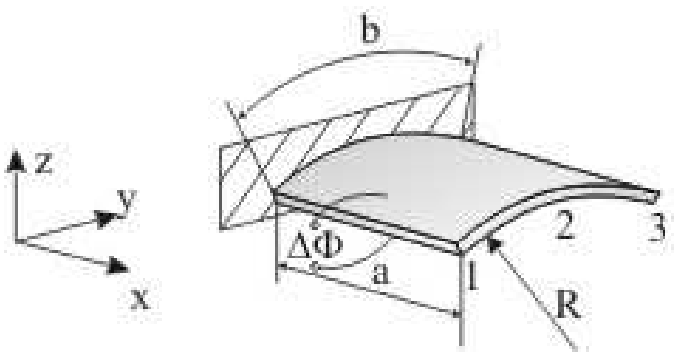

Fig. 3. Clamped cylindrical active shell and electric excitation

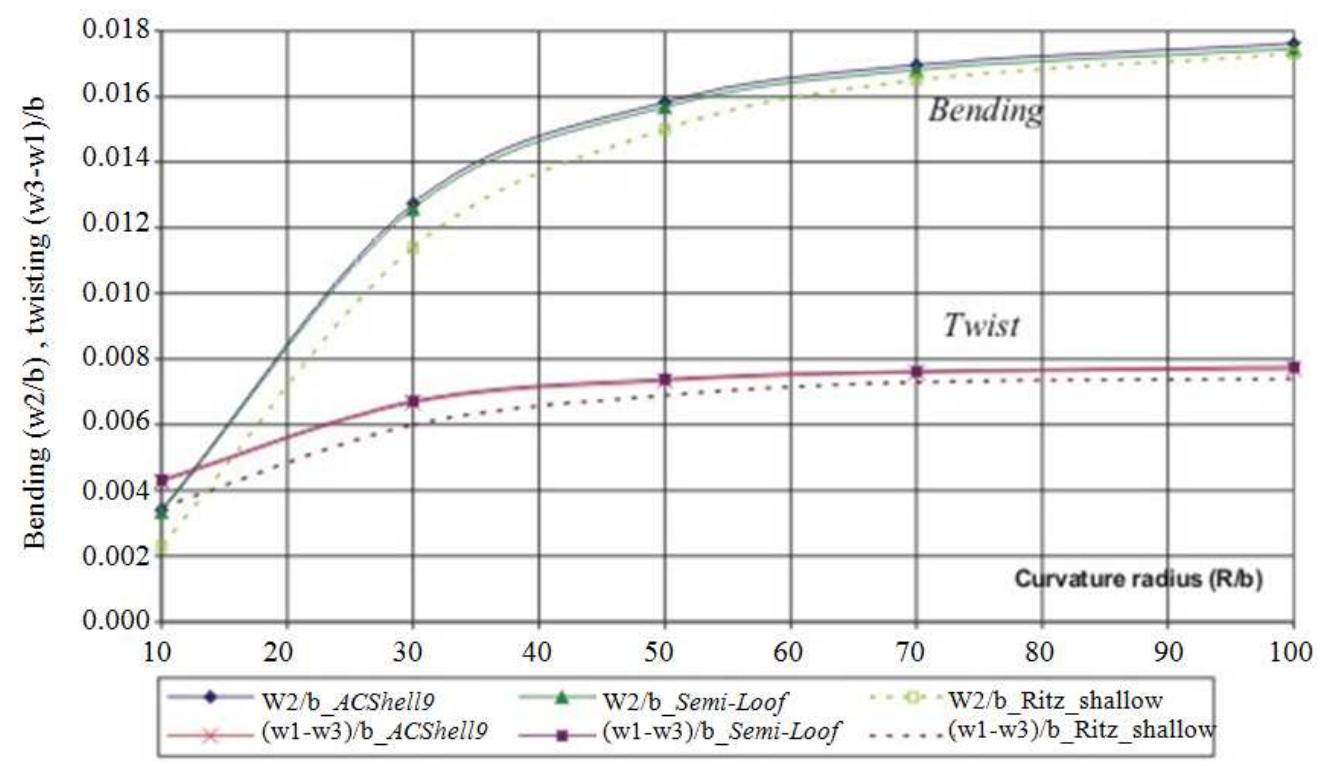

Fig. 4. Shape control of the simply supported composite active plate 


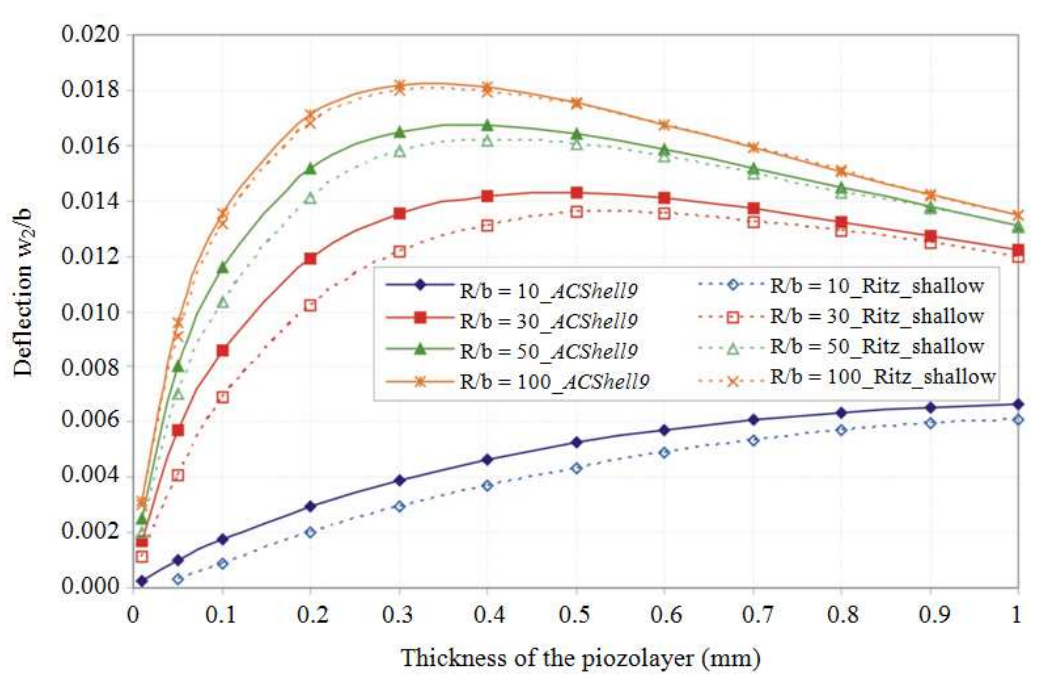

Fig. 5. Effect of the piezoelectric layer thickness on the shell bending

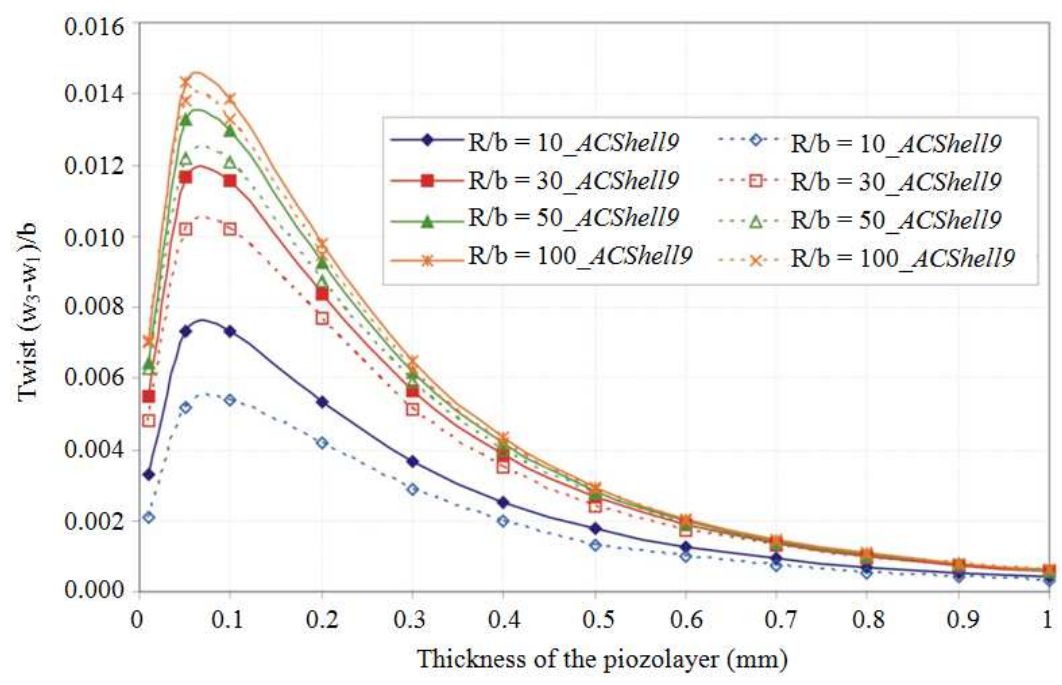

Fig. 6. Effect of the piezoelectric layer thickness on the shell twist

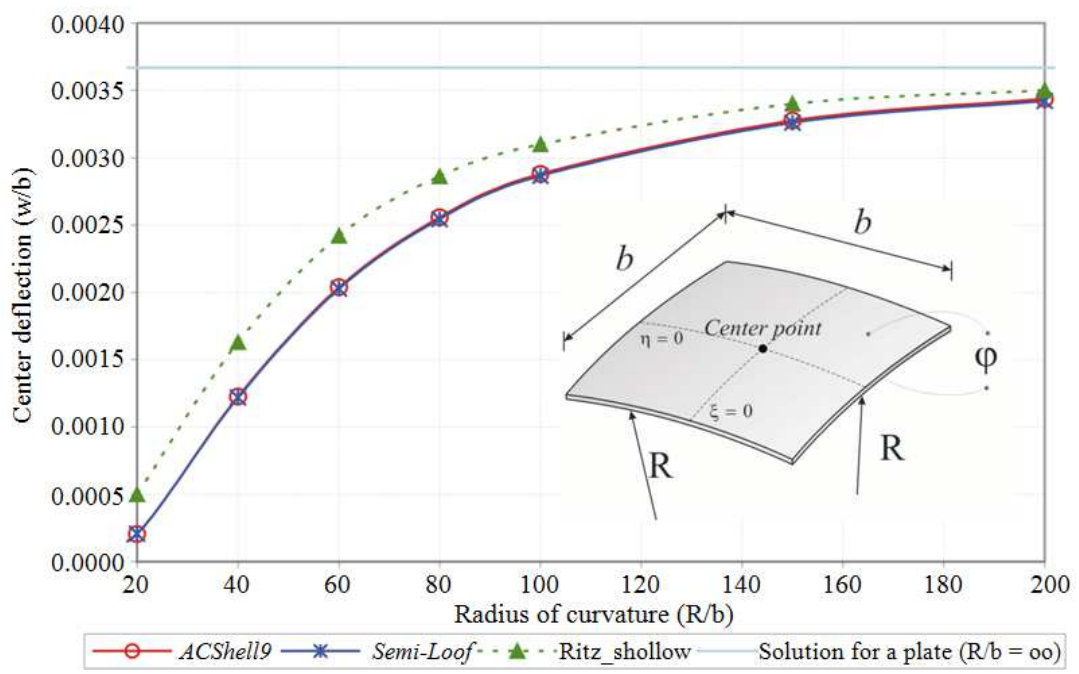

Fig. 7. Effect of the piezoelectric layer thickness on the normalized shell deflection 
The influence of the piezoelectric layer thickness on the resulting bending and twist of the shell under a uniform electric field of $\mathrm{E}=400 \mathrm{Vmm}^{-1}$ has also been investigated. The obtained results are summarized in Fig. 5 and 6. Both diagrams show that the shell curvature considerably reduces the efficiency of the piezoelectric actuation. They also reveal the existence of optimal piezolayers thickness with respect to the objective of achieving maximal deformation. Considering bending, the optimal thickness for maximal deflection decreases together with the curvature, i.e. as the shell tends to a flat structure. On the other hand, the optimal thickness for the twist is hardly affected by the shell curvature, but as the piezolayer thickness approaches the optimal values the induced twist becomes more affected by the shell curvature than at other values of the thickness.

\section{Simply Supported Active Spherical Shell}

A simply supported spherical shell is considered in the last example. The sequence of fiber-reinforced layers, $[0 / 90 / 0]_{\mathrm{S}}$, is now balanced. The excitation is achieved in exactly the same manner as in the previously considered cases (voltage of $\varphi=100 \mathrm{~V}$, Fig. 7) and the transverse deflection of the structure's mid-point is observed. The finite element results are obtained with the same $8 \times 8$ mesh and the results for different radii $\mathrm{R}$ are given in the Fig. 7. One may note that the Ritz solution yields higher values for displacements compared to the FEM solution. This is again a consequence of the deficiency of the approximation functions used by Kioua and Mirza (2000) for the Ritz solution. The Ritz solution was based on polynomials up to the second order, whereas some of the solutions analyzed above require the polynomials of at least fourth order. In those cases, the Ritz solution yields the best possible approximation of the actual deformation that can be formed with the applied approximation functions. How the approximation actually performs depends on the point of the structure where the result is evaluated. The above examples have demonstrated that the Ritz solution may underestimate but also overestimate the actual result. In the case of plate, the Ritz solution even yielded recovery of the initial, flat geometry, which is virtually impossible under the given loads-surface pressure and distributed edge bending moments induced through the piezoelectric effect.

\section{Conclusion}

In vibration and noise control as well as in shape control of adaptive structures, commercially available piezoelectric patches are very common active elements. They are used both as actuators and sensors.
The actuator function was demonstrated in this paper. For the analysis and design process of smart structures with integrated piezoelectric patches, the finite element method provides an effective simulation approach. The paper presented the developed shell finite element that can be gainfully used for modeling and simulation of the behavior of thin-walled hybrid composite active structures. Application of the developed ACShell9 element was demonstrated on a several static examples, while the comparison with the already existing and tested Semi-Loof shell element has proven the accuracy offered by the ACShelly element. The results from the two elements are obviously in very high agreement. On the other side, discrepancies between the finite element and the Ritz solutions were noted. They are explained in the paper, thus confirming the well-known advantages of the finite element approach with respect to the conventional Ritz method.

\section{Authors' Contribution}

DM: Development of the applied shell type finite element, integration of the developed element into an existing solver, choice of the verifying examples, writing of the manuscript.

MZ: Integration of the developed element into an existing solver, choice of the verifying examples, writing of the manuscript.

\section{Ethics}

The authors would like to disclose that Dr. Dragan Marinkovic (the first author) is a member of the editorial board for the American Journal of Engineering and Applied Sciencs.

\section{References}

Ahmad, S., B.M. Irons and O.C. Zienkiewicz, 1970. Analysis of thick and thin shell structures by curved finite elements. Int. J. Numerical Meth. Eng., 2: 419-451. DOI: 10.1002/nme. 1620020310

Benjeddou, A., 2000. Advances in piezoelectric finite element modeling of adaptive structural elements: A survey. Comput. Structures, 76: 347-363. DOI: 10.1016/S0045-7949(99)00151-0

Kioua, H. and S. Mirza, 2000. Piezoelectric induced bending and twisting of laminated composite shallow shells. Smart Mater. Structures, 9: 476-484. DOI: 10.1088/0964-1726/9/4/310

Marinkovic, D., 2007, A new finite composite shell element for piezoelectric active structures. Ph.D. Thesis, Otto-von-Guericke Universität Magdeburg. 
Marinkovic, D., H. Köppe and U. Gabbert, 2008. Degenerated shell element for geometrically nonlinear analysis of thin-walled piezoelectric active structures. Smart Mater. Structures, 17: 015030-015030. DOI: 10.1088/0964-1726/17/01/015030

Marinkovic, D., M. Zehn and Z. Marinković, 2013. The analysis of FEM results convergence in modelling piezoelectric active shell structures. Trans. FAMENA, 37: 17-27.

Marinkovic, D., Z. Marinković and G. Petrović, 2012. On efficiency of a single-layer shell element for composite laminated structures. Facta Universitatis Series Mechanical Eng., 10: 105-112.
Nestorovic, T., S. Shabadi, D. Marinković and M. Trajkov, 2013. Modeling of piezoelectric smart structures by implementation of a user defined shell finite element, Facta Universitatis Series Mechanical Eng., 11: 1-12.

Nestorovic, T., D. Marinković, S. Shabadi and M. Trajkov. 2014. User defined finite element for modeling and analysis of active piezoelectric shell structures. Meccanica, 49: 1763-1774. DOI: $10.1007 / \mathrm{s} 11012-014-9925-\mathrm{x}$

Seeger, F., H. Köppe and U. Gabbert, 2001. Comparison of different shell elements for the analysis of smart structures. ZAMM, 81: 887-888. 